\title{
Network Requirement for Management of Multimedia over Wireless Channel
}

\author{
Bing Zheng ${ }^{1}$ and Mohammed Atiquzzaman ${ }^{2}$ \\ 1 New Focus Inc \\ 5215 Hellyer Ave., San Jose, CA 95138. \\ Email:zhengbin@ieee.org \\ 2 School of Computer Science \\ University of Oklahoma, Norman, OK 73019-6151. \\ Email: atiq@ou.edu
}

\begin{abstract}
Wireless channels have a high channel bit error rate and limited bandwidth. The high bit error rate degrades the quality of multimedia transmission, and also increases the buffer size requirement at the client. In this paper, we propose a novel selective retransmission scheme for multimedia transmission over wireless ATM networks using the ABR service. Our objective is to develop a simple cost effective scheme which offers quality of service for MPEG compressed video transmitted over a noisy wireless channel. We have developed an analytical model to determine the networking requirements and receiver buffer size for our proposed scheme for transmitting multimedia over wireless ATM.
\end{abstract}

\section{Introduction}

The transmission of multimedia over wireless channel is becoming a research topic of growing interest 123 . With the emergence of next generation high speed wireless ATM networks, it is expected that multimedia will be the main source of traffic in future wireless ATM networks. Wireless transmissions use radio as the transmission media which is easily affected by environments such as buildings, moving objects, and atmosphere, etc., giving rise to issues such as effective bandwidth allocation.

The ATM Forum has standardized four service classes: Constant Bit Rate (CBR), Variable Bit Rate (VBR) including real time VBR and non-real time VBR, Available Bit Rate (ABR) and Unspecified Bit Rate (UBR). CBR has the highest quality of service guarantee and the highest service cost. However, ABR offers cost effective service at an acceptable quality of service, flexible bandwidth allocation, and has a high utilization of network bandwidth. We will therefore, investigate the effectiveness of using the ATM ABR service to transport multimedia over a wireless ATM network. By combining the advantages of ATM and wireless, it is possible to implement a wireless ATM network which will offer high speed, quality of service, and mobility to future multimedia users [456].

Networked multimedia applications include remote education, video-ondemand, tele-shopping, home game entertainment, etc. Because of traffic characteristics such as high bit rate, video will be the dominant traffic in multimedia

The original version of this chapter was revised: The copyright line was incorrect. This has been corrected. The Erratum to this chapter is available at DOI: 10.1007/978-3-540-45812-8_28

K.C. Almeroth and M. Hasan (Eds.): MMNS 2002, LNCS 2496, pp. 49-61, 2002.

(C) IFIP International Federation for Information Processing 2002 
streams, and hence needs to be managed efficiently [7. Video must be compressed (for example, MPEG) to reduce the required transmission rate. In contrast to data, multimedia can tolerate a certain level of error. Therefore, although a wireless network has a high bit error rate when compared to a wireline network, it is possible to cost effectively transmit multimedia over wireless networks with acceptable quality of service (QoS).

In their experimental studies on effective algorithms for video transmission over a wireless channel [8], the authors studied the go-back-W retransmission scheme with window size W. They also considered the receiver buffer size for single retransmission. Their experimental results have shown that retransmission without FEC can improve the video transmission quality. However, their studies did not present detailed theoretical model and analysis of their retransmission scheme. Since the effectiveness of a retransmission scheme depends on the network parameters such as channel bit error rate, channel transmission rate, and video parameters such as the video frame size, it is very important to study the relationship between these parameters and the effectiveness and efficiency of retransmission schemes. Moreover, to sustain continuous video display at the receiver considering transmission over a noisy channel, continuous monitoring of the network status is required to prevent transmission collapse. Finally, during the time taken for retransmission, the receiver can only decode the buffered data upto the errored frame. To prevent starvation at the receiver (resulting in frozen display), the receiver buffer must have a minimum fill level to sustain continuous display during retransmission. The minimum fill level thereafter decides the minimum receiver buffer size. The longer the retransmission time, the larger the minimum fill level. Since the retransmission time depends on the channel bit error rate, the minimum receiver buffer size depends on the channel bit error rate. The authors in 9] have reviewed several error resilient video coding methods for MPEG-2 transmission over wireless ATM networks. They found that in a WAN testbed, the cell losses and wireless packet losses occur independently.

The high bit error rate of wireless channels raises a number of challenging issues for multimedia transmission over a wireless ATM network. Such issues include techniques to ensure a continuous display at the receiver in spite of errors in the received video. Currently, errors are compensated mainly by Forward Error Correction (FEC) which employs redundant bits to passively correct errors at the receiver. Although FEC can correct some errors without retransmission, it needs extra bandwidth to transmit a lot of redundant bits for correction. This lowers the utilization of bandwidth which is expensive for wireless channels. Furthermore, implementing FEC requires a lot of computing power at the receiver, thereby increasing the cost of the receiver. Additionally, complicated error concealment algorithms will have to be implemented at the receiver for different error types [10. All of the above issues may prevent cost effective wide scale commercial deployment of wireless multimedia receiver if FEC is used at the receiver. Therefore, new schemes for error correction at the receiver are required for widespread deployment of cost effective multimedia transmission over noisy wireless links. 
In this paper, a new selective retransmission scheme is proposed for multimedia transmission over noisy wireless channels in order to ensure acceptable video quality at the receiver and allow the design of a cost effective receiver. Since multimedia can tolerate a certain level of error with little effect on picture quality, our scheme sets a threshold on the number of errored cells, and to decide whether retransmission is required. To prevent the receiver from starvation (i.e. ensuring a continuous display), a receiver buffer is required.

Our objective is to develop an efficient error handling scheme for wireless transmission of multimedia, and to show that the scheme results in a cost effective wireless receiver. The significance of our scheme is that it does not require powerful computing capability and complicated error correction algorithm to be implemented at the wireless receiver. The wireless receiver will require simple hardware and thereby reduce the receiver cost. Our scheme also offers a method to monitor the status of the transmission system to check if the conditions to maintain transmission are satisfied. Our performance measure is maintaining a continuous display at the wireless receiver.

The rest of the paper is organized as follows. In Section [2, we propose the system stack model and our proposed selective retransmission scheme. Analysis of a multimedia wireless system using our proposed scheme is performed in Section 3. In Section 4, the minimum receiver buffer size is derived. Numerical results obtained from our analysis are given in Section $\mathbf{5}$, followed by conclusions in Section [6.

\section{System Model and Proposed Retransmission Scheme}

In this section, we describe the system model and the operating principle for our selective retransmission scheme. It will be used in Section 3 to derive values of network parameters and the size of receiver buffer to sustain continuous display at the receiver. As shown in Figure 1, the system consists of a multimedia server and receivers connected by a wireless ATM network using the ATM ABR service. The protocol stack consists of four layers as shown in Figure 2.

MPEG compressed video consists of I, P and B frames. The frames are grouped to form a special structure called Group of Picture (GoP). Each GoP includes an I frame followed by a number of $\mathrm{P}$ and $\mathrm{B}$ frames. A GoP is denoted by $M m N n$ which represents a total of $n$ frames in the GoP with $m-1$ number of $\mathrm{B}$ frames between the $\mathrm{I} / \mathrm{P}$ or $\mathrm{P} / \mathrm{P}$ frames. At the decoder, error in a received I frame will affect all the frames in the GoP, while error in a received $\mathrm{P}$ frame will affect the quality of the subsequent $\mathrm{P}$ and $\mathrm{B}$ frames. An error in a received B frame only affects its own quality. Therefore, I frame is the most important, while the B frame is the least important. To maintain the quality of video at the receiver, we propose the following retransmission scheme based on errors in the received video stream at the receiver.

- If the number of errored cells in an I frame is higher than an acceptable error threshold for the I frame, the cells belonging to the I frame are discarded, and the I frame is retransmitted from the multimedia server; 


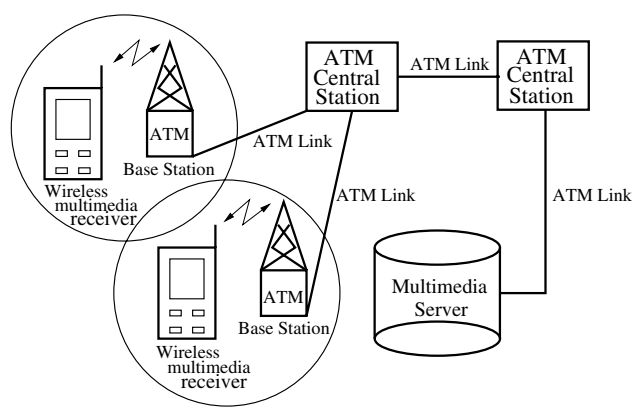

Fig. 1. The element of wireless ATM network for multimedia application.

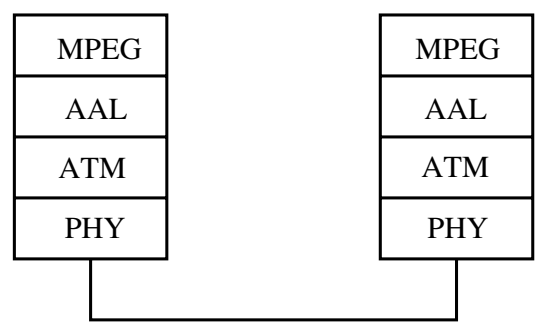

Fig. 2. The protocol stack for MPEG video transmission over ATM wireless networks.

- If the number of errored cells in a $\mathrm{P}$ frame is higher than the acceptable error threshold for the $\mathrm{P}$ frame, the $\mathrm{P}$ frame is discarded and is retransmitted;

- Errored cells in B frames are discarded and are not retransmitted.

In the next section, we develop a mathematical model to analyze our proposed scheme, to derive the network requirements to sustain a continuous video display at the receivers, and to determine the minimum size of the receiver buffer. In our discussion, we assume:

- The wireless channel has a bit error rate which is independent of the traffic pattern and the channel transmission rate;

- Each bit has equal probability of being errored during transmission. Moreover, each cell has equal probability of having errors;

- The channel has fixed round trip time between the server and receiver;

- The ATM ABR service is used to transmit multimedia [7], i.e. the amount of bandwidth available to the application changes over time depending on the network congestion level.

\section{System Analysis}

In this section, we develop a mathematical model to analyze our proposed retransmission scheme. First, we define the following variables.

\subsection{Notation}

$\rho$ : Channel bit error rate of the wireless link;

$S_{I}, S_{P}$ and $S_{B}$ : Average size of I, P and B frames respectively of MPEG video; $N$ : Number of bits in the payload of a cell (For an ATM cell, $N=48^{*} 8$ );

$N_{I}, N_{P}$ and $N_{B}$ : Average number of cells belonging to I, $\mathrm{P}$ and $\mathrm{B}$ frame in a GoP; 
$d_{I}, d_{P}$ : Threshold for the acceptable number of errored cells in I and $\mathrm{P}$ frames respectively;

$p$ : Probability that a cell has error;

$W_{I}$ : Probability that an I frame is errored given that an error has occurred;

$W_{P}$ : Probability that a $\mathrm{P}$ frame is errored given that an error has occurred;

$P_{I}\left(d_{I}\right)$ : Probability that an I frame needs to be retransmitted, called $I$ frame transmission failure probability;

$P_{P}\left(d_{P}\right)$ : Probability that a $\mathrm{P}$ frame needs to be retransmitted, called $P$ frame transmission failure probability;

$T_{d}$ : Fixed round trip time (FRTT) from server to receiver;

$T_{r}$ : Average successful retransmission time for an errored frame, called the average system recover time interval. This is the average time required for successful retransmission;

$\tau_{f}$ : Average time interval between consecutive frame errors, called average system failure time interval;

$\lambda$ : Channel transmission rate;

$\mu(t)$ : Multimedia playback rate at time $t$;

$C_{m i n}$ : Minimum receiver buffer size.

\subsection{Average System Failure and Recover Time}

Since the probability of a bit being in error is $\rho$, the probability that the payload of a cell has no error is $(1-\rho)^{N}$. Therefore, the probability $p$ that a cell is in error can be expressed as:

$$
p=1-(1-\rho)^{N}
$$

The probability $P_{I}\left(d_{I}\right)$ that an I frame needs to be retransmitted is the probability that the number of errored cells in the frame is higher than the threshold $d_{I}$. Therefore, $P_{I}\left(d_{I}\right)$ can be expressed as:

$$
P_{I}\left(d_{I}\right)=1-\sum_{i=0}^{d_{I}}\left(\begin{array}{c}
N_{I} \\
i
\end{array}\right) p^{i}(1-p)^{N_{I}-i}
$$

where $\sum_{i=0}^{d_{I}}\left(\begin{array}{c}N_{I} \\ i\end{array}\right) p^{i}(1-p)^{N_{I}-i}$ represents the probability of the I frame having upto $d_{I}$ errored cells. Similarly, $P_{P}\left(d_{P}\right)$ can be expressed as:

$$
P_{P}\left(d_{P}\right)=1-\sum_{j=0}^{d_{P}}\left(\begin{array}{c}
N_{P} \\
j
\end{array}\right) p^{j}(1-p)^{N_{P}-j}
$$

Given that a frame is in error, the probability that the frame is an I frame is proportional to the size of the I frame in a GoP. Therefore, $W_{I}$ can be expressed as:

$$
W_{I}=\frac{S_{I}}{S_{I}+S_{P}\left(\frac{n}{m}-1\right)+S_{B} \frac{m-1}{m} n}
$$


where the denominator represents the size of a GoP. Similarly, we can obtain the expression for $W_{P}$ as:

$$
W_{P}=\frac{S_{P}}{S_{I}+S_{P}\left(\frac{n}{m}-1\right)+S_{B} \frac{m-1}{m} n}
$$

If an I frame is in error, the average time $T_{r}^{I}$ for successful retransmission is expressed as:

$$
\begin{aligned}
T_{r}^{I} & =\left(T_{d}+\tau_{s}^{I}\right)\left(1-P_{I}\left(d_{I}\right)\right)+2\left(T_{d}+\tau_{s}^{I}\right) P_{I}\left(d_{I}\right)\left(1-P_{I}\left(d_{I}\right)\right)+\ldots \ldots \\
& =\frac{T_{d}+\tau_{s}^{I}}{1-P_{I}\left(d_{I}\right)}
\end{aligned}
$$

where $\tau_{s}^{I}=\frac{S_{I}}{\lambda}$ is the time required to transmit an I frame into the wireless channel. Similarly, $T_{r}^{P}$ for successful retransmission of a $\mathrm{P}$ frame can be expressed as:

$$
T_{r}^{P}=\frac{T_{d}+\tau_{s}^{P}}{1-P_{P}\left(d_{P}\right)}
$$

where $\tau_{s}^{P}=\frac{S_{P}}{\lambda}$ is the time to transmit a $\mathrm{P}$ frame into the channel.

Therefore, the average system recover time $\left(T_{r}\right)$ is the statistical sum of the I and $\mathrm{P}$ frame recover times $T_{r}^{I}$ and $T_{r}^{P}$ with weights $W_{I}$ and $W_{P}$ respectively. Therefore, $T_{r}$ can be expressed as:

$$
T_{r}=T_{r}^{I} W_{I}+T_{r}^{P} W_{P}
$$

Because a wireless channel has significant error rate, let's say that an error happens after time $\tau_{f}$ which is defined as the average system failure time. Since the acceptable error thresholds are $d_{I}$ and $d_{P}$ for I and $\mathrm{P}$ frames respectively, the average system failure time is:

$$
\tau_{f}=\frac{\min \left(d_{I}, d_{P}\right)}{\lambda \rho}
$$

where the $\lambda$ is the available bandwidth of the ABR connection.

An interactive receiver can perform VCR like interactive functions such as stop, playback, fastforward (FFW), and fastbackward (FBW) which are represented by a state transition diagram as shown in Figure 3 . In the FFW/FBW state, the receiver will consume data at a higher speed than the playback state. Therefore, in response to a FFW/FBW request, the sever will request higher bandwidth than is required in normal playback. The bandwidth $\lambda$ required by the server is decided by the level of interactivity of the receiver and the available bandwidth.

Let's denote the stop, playback, FFW and FBW states of the receiver by $0,1,2,3$, and the state transition probability from state $i$ to state $j$ (where $0 \leq i, j \leq 3)$ is represented by $\theta_{i j}$. If stationary state probability vector for the 
receiver is $X=\left(X_{0}, X_{1}, X_{2}, X_{3}\right), X$ can be obtained by solving the following equation [1]:

$$
\begin{aligned}
& X_{0}=\frac{1}{1+\theta_{0,1} / \theta_{1,0}\left(1+\theta_{1,2} / \theta_{2,1}+\theta_{1,3} / \theta_{3,1}\right)} \\
& X_{1}=\frac{\theta_{0,1}}{\theta_{1,0}\left(1+\theta_{0,1} / \theta_{1,0}\left(1+\theta_{1,2} / \theta_{2,1}+\theta_{1,3} / \theta_{3,1}\right)\right)} \\
& X_{2}=\frac{\theta_{0,1} \theta_{1,2}}{\theta_{1,0} \theta_{2,1}\left(1+\theta_{0,1} / \theta_{1,0}\left(1+\theta_{1,2} / \theta_{2,1}+\theta_{1,3} / \theta_{3,1}\right)\right)} \\
& X_{3}=\frac{\theta_{0,1} \theta_{1,3}}{\theta_{1,0} \theta_{3,1}\left(1+\theta_{0,1} / \theta_{1,0}\left(1+\theta_{1,2} / \theta_{2,1}+\theta_{1,3} / \theta_{3,1}\right)\right)}
\end{aligned}
$$

For interactive multimedia, the expected channel transmission rate $E[\lambda(t)]$ is therefore expressed as the statistical sum of the playback rate, fastforward rate, and fastbackward rate with weights $X_{1}, X_{2}$, and $X_{3}$.

$$
E[\lambda(t)]=\left[X_{1}+k\left(X_{2}+X_{3}\right)\right] \mu(t)
$$

where $k$ is the $\mathrm{FFW} / \mathrm{FBW}$ speed factor implying that the channel transmission rate is $k$ times that of the normal playback rate.

\subsection{System Model and Dynamics}

If the receiver receives a frame with the number of errored cells exceeding the threshold, the server will retransmit the corresponding frame by switching to the retransmission state. Therefore, the status of the system can be divided into two states as shown in Figure 4 :

- State 0: the normal state in which multimedia is continuously received at the receiver;

- State 1: the retransmission state in which the system is retransmitting a frame. The receiver consumes data upto the errored frame and then waits until the successful arrival of the retransmitted frame.

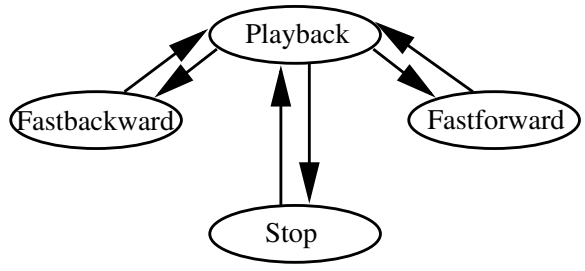

Fig. 3. The interactive operation state at receiver.

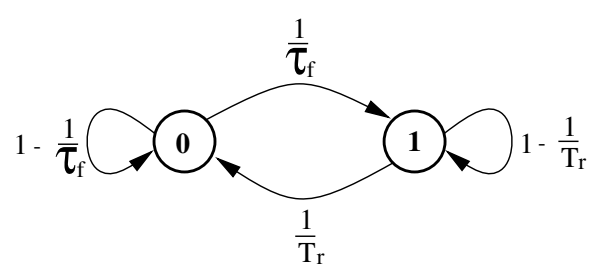

Fig. 4. The state diagram of the serverclient type wireless multimedia transmission system. 
From Figure 4, the state transition matrix $M$ is expressed as:

$$
M=\left[\begin{array}{cc}
1-\frac{1}{\tau_{f}} & \frac{1}{\tau_{f}} \\
\frac{1}{T_{r}} & 1-\frac{1}{T_{r}}
\end{array}\right]
$$

Let $V=\left(V_{0}, V_{1}\right)$ denote the long term stationary state vector, which satisfies the stationary equation:

$$
V=V M
$$

By solving the stationary equation, the steady state probabilities are obtained as:

$$
\begin{aligned}
& V_{0}=\frac{1}{1+\alpha} \\
& V_{1}=\frac{\alpha}{1+\alpha}
\end{aligned}
$$

where $\alpha=\frac{T_{r}}{\tau_{f}}$. In order for the system to work satisfactorily, it should be at state 0 most of the time, i.e., $\alpha<<1$. This implies:

$$
\frac{T_{d}+\tau_{s}^{I}}{1-P_{I}\left(d_{I}\right)} W_{I}+\frac{T_{d}+\tau_{s}^{P}}{1-P_{P}\left(d_{P}\right)} W_{P} \ll \frac{\min \left(d_{I}, d_{P}\right)}{\lambda \rho}
$$

If $\operatorname{Det}(M)=0$, there is no non-trivial steady state solution, i.e., the system does not have any long term stationary state. In this case, the system will not work properly; this gives the critical condition that the system will be down:

$$
T_{r} \tau_{f}=T_{r}+\tau_{f}
$$

Because $T_{r}$ and $\tau_{f}$ are related to channel bit error rate, channel transmission rate, multimedia frame size, GoP structure, acceptable error threshold, FRTT, etc., by monitoring and dynamically adjusting these parameters during multimedia transmission, we can satisfy acceptable quality of service and prevent the video transmission from collapse. For example, ATM ABR service offers the possibility of adjusting channel transmission rate during transmission by requesting bandwidth using the Resource Management (RM) cell.

\section{Minimum Receiver Buffer Requirement}

For MPEG video, the decoding of $\mathrm{P}$ and $\mathrm{B}$ frames in a GoP depends on the I frame in that GoP; the decoding of a $\mathrm{B}$ frame depends only on the $\mathrm{P}$ frames preceding and following the $\mathrm{B}$ frame. Therefore, an error in an $\mathrm{I}$ or $\mathrm{P}$ frame propagates to all other frames in the GoP. The decoder at the receiver will deplete the buffered data until the damaged frame is recovered. Assuming that the system failed at time $t_{1}$, to sustain a continuous display at receiver, the amount of buffered data $C\left(t_{1}\right)$ must satisfy:

$$
C\left(t_{1}\right)=\int_{t_{1}}^{t_{1}+T_{r}} \mu(t) d t
$$


By using the average expression, let $E\left[\mu\left(t_{1}\right)\right]$ denote the average value of $\mu(t)$ for $t_{1} \leq t \leq t_{1}+T_{r}$. Then $C\left(t_{1}\right)$ can be expressed as

$$
C\left(t_{1}\right)=T_{r} E\left[\mu\left(t_{1}\right)\right]
$$

The minimum amount of buffered data also sets the minimum receiver buffer size $C_{\min }$ :

$$
C_{\text {min }}=T_{r} E\left[\mu\left(t_{1}\right)\right]
$$

By substituting the expression for $T_{r}$ into Equation (20), we get:

$$
C_{\text {min }}=\left(\frac{T_{d}+\tau_{s}^{I}}{1-P_{I}\left(d_{I}\right)} W_{I}+\frac{T_{d}+\tau_{s}^{P}}{1-P_{P}\left(d_{P}\right)} W_{P}\right) E\left[\mu\left(t_{1}\right)\right]
$$

From Equation (21), the minimum receiver buffer size is decided by the playback rate, the FRTT, the link rate, and the frame failure probability.

\section{$5 \quad$ Numerical Results}

In this section, we present results to evaluate the performance of our proposed scheme and show its effectiveness in multimedia transmission over wireless ATM. The average system failure time interval versus the level of user interactivity is shown in Figure 5. As expected from Equations (9) and (11), with an increase in the fastforward/fastbackward probability of the user, the required channel transmission rate also increases. This results in more data bits being transmitted in unit time, i.e. higher probability of error happening in unit time. Therefore, the average system failure time interval decreases.

The average system failure time interval versus the channel bit error rate is shown in Figure [6. As pointed out in Equation (9), the average system failure time interval is inversely proportional to the channel bit error rate.

The average system failure time interval versus the cell error threshold is shown in Figure [7. The average system failure time interval linearly increases with an increase in the cell error threshold which depends on the QoS acceptable by the user.

The I frame and $\mathrm{P}$ frame failure probability versus channel bit error rate is shown in Figure 8. As the channel bit error rate increase, the frame failure probability increase abruptly. Since the size of I frame is much larger than that of $\mathrm{P}$ frame, the I frame failure probability is much more sensitive to the channel bit error rate than the $\mathrm{P}$ frame.

The I frame and $\mathrm{P}$ frame failure probabilities versus cell error threshold are shown in Figure 9]. As the cell error threshold increase, the frame failure probability decrease abruptly. This enables us to properly choose the cell error threshold to compensate for the the channel bit error rate which in turn reduces the number of retransmissions and increases the network bandwidth utilization.

The average system recover time interval versus channel bit error rate is shown in Figure [0] When channel error rate is relatively small, the average 


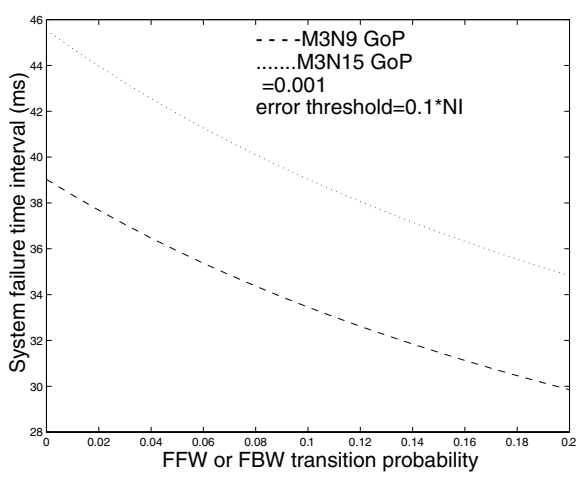

Fig. 5. Average system failure time interval versus the receiver's level of interactivity.

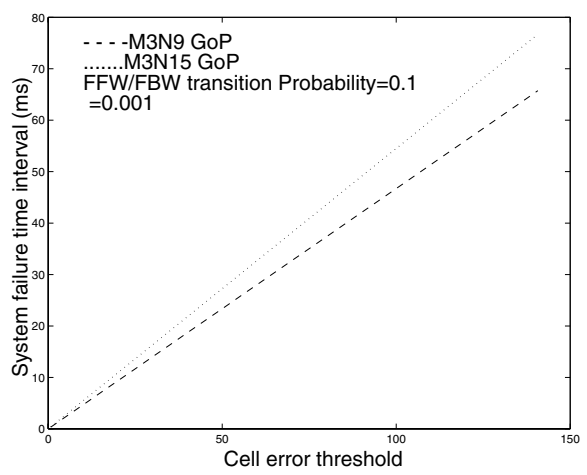

Fig. 7. Average system failure time interval versus cell error threshold.

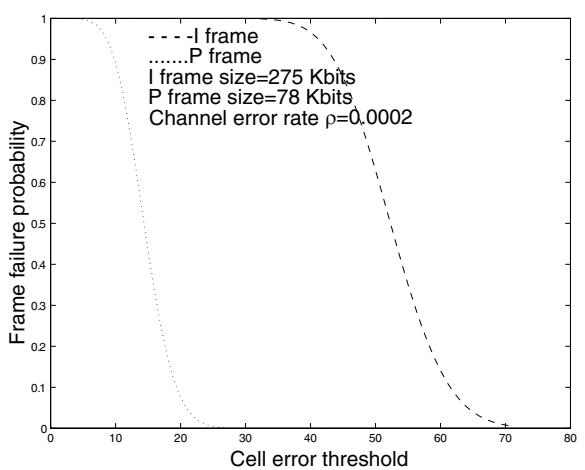

Fig. 9. I \& P frame failure probability versus the cell error threshold.

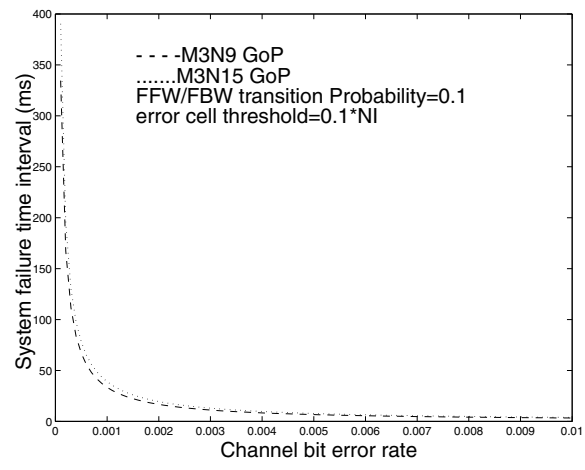

Fig. 6. Average system failure time interval versus the channel bit error rate.

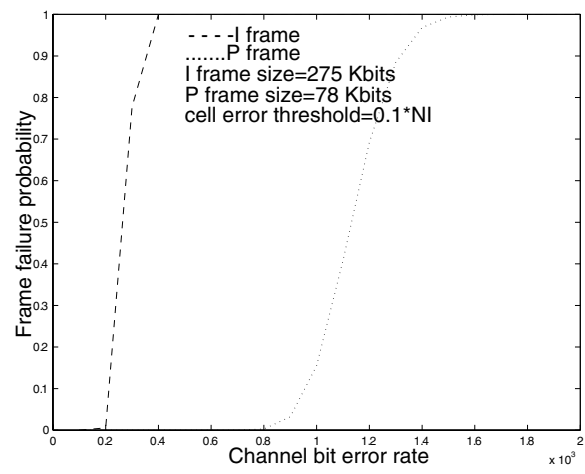

Fig. 8. I \& P frame failure probability versus channel bit error rate.

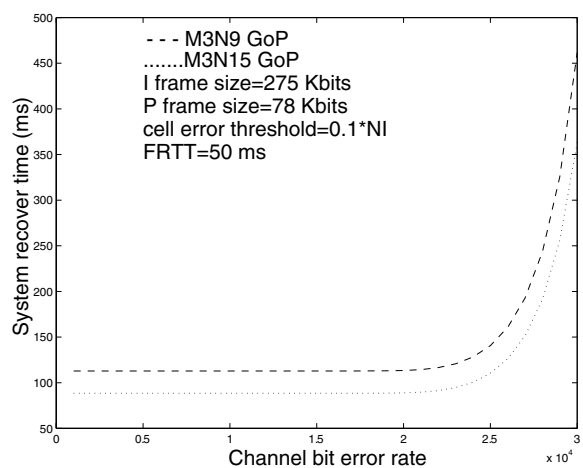

Fig. 10. Average system recover time interval versus channel bit error rate. 
retransmission time is almost constant. As the channel bit error rate increases to some area, the average retransmission time increases sharply.

The system recover time interval versus receiver's level of interactivity is shown in Figure [I]. As the level of interactivity increases, the transmission rate increases, the time to inject a frame into network decrease, and the average retransmission time decreases.

The average system recover time interval versus the I frame size is shown in Figure 12] As the I frame size increases, the probability of I frame transmission increases with an associated increase in time to inject an I frame into network. Therefore, the average retransmission time will increase.

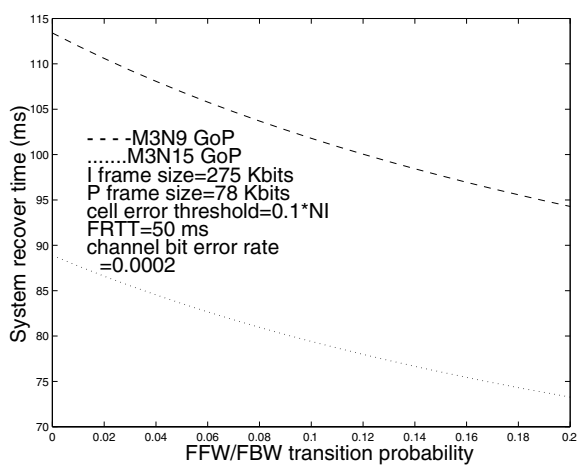

Fig. 11. Average system recover time interval versus user's level of interactivity.

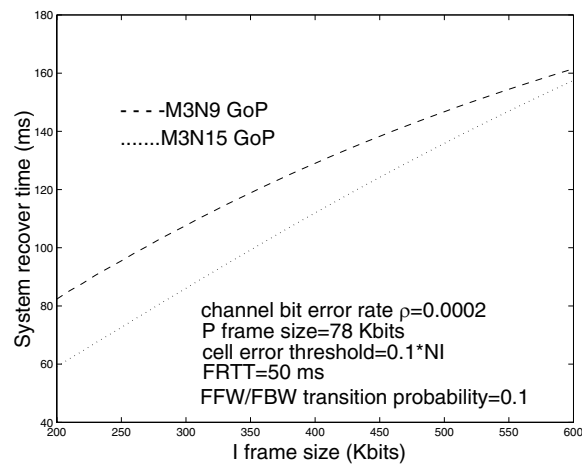

Fig. 12. Average system recover time interval versus I frame size.

The minimum receiver buffer size versus channel bit error rate is shown in Figure 13, As described in Equation (201), the minimum receiver buffer size is proportional to the average system retransmission time. Therefore, when the channel error rate is low, the minimum receiver buffer size is almost constant. As the channel bit error rate increases, the minimum receiver buffer size increases abruptly in order to maintain a continuous display at the receiver.

The minimum receiver buffer size versus the user's level of interactivity is shown in Figure 14. Since interactivity only affects the channel transmission rate, its effect on receiver buffer size is relatively small.

The minimum receiver buffer size versus the I frame size is shown in Figure 15. As the I frame size increases, the frame transmission failure probability increases, the time needed to inject the frame into the network increases, and the required channel transmission rate increases resulting in an increase in the minimum receiver buffer size as shown in Equation (21). 


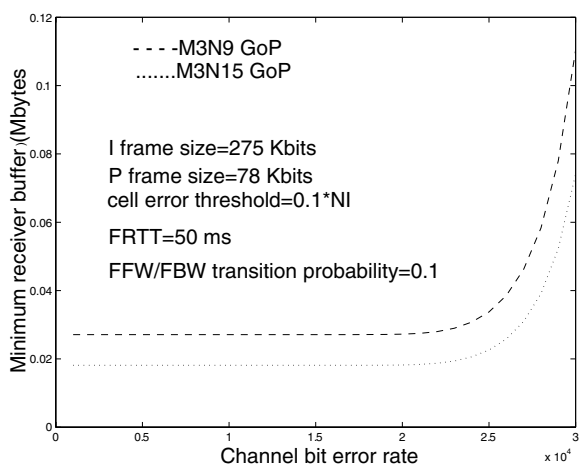

Fig. 13. Minimum receiver buffer size versus channel bit error rate.

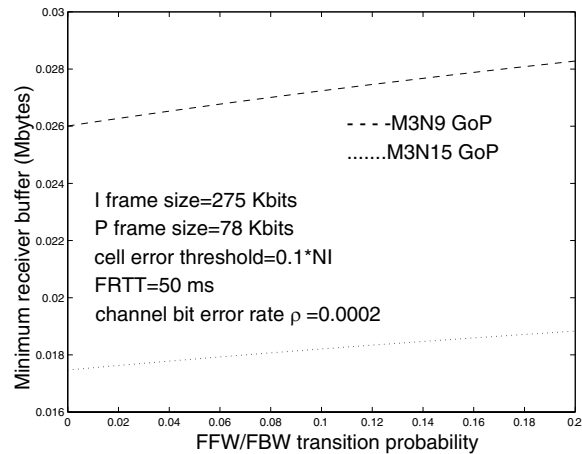

Fig. 14. Minimum receiver buffer size versus receiver's level of interactivity.

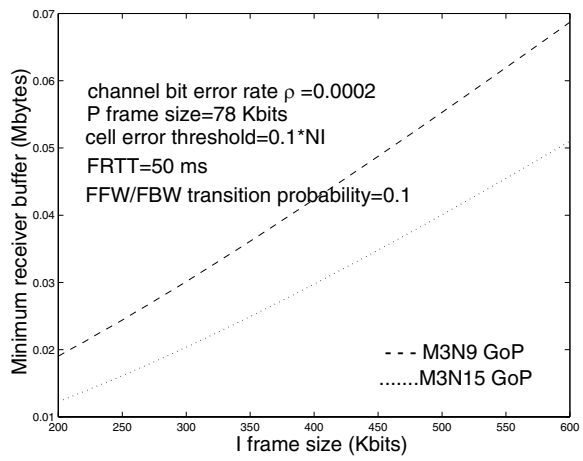

Fig. 15. Minimum receiver buffer size versus I frame size. 


\section{Conclusions}

In this paper, we have proposed a new selective retransmission scheme for multimedia transmission over noisy wireless channel using the ATM ABR service. We analyzed the system requirements and minimum receiver buffer size for providing acceptable QoS to the user. We conclude that the average system failure time interval decreases with an increase in the channel error rate and level of user interactivity. The I frame has a much higher transmission failure probability than the $\mathrm{P}$ frame for a given channel bit error rate.

The average system recover time interval has tight relationship with the channel bit error rate and the frame sizes of video, but has little effect on the level of user interactivity. By choosing an acceptable cell error threshold for a given channel error rate, our proposed selective retransmission scheme requires a small receiver buffer to cost effectively transmit multimedia over a wireless channel with a bit error rate less than $10^{-4}$.

Our investigation on the effect of coding on the performance of our proposed algorithm shows that for multimedia over a noisy wireless channel, a large GoP structure has a better performance than a small GoP structure.

\section{References}

1. Lombardo, A., Palazzo, S., Schenbra, G.: Management of wireless ATM networks loaded by mobile ABR source traffic. In: Globecom, Brazil (1999) 2758 - 276249

2. Kwok, Y.K., Lau, V.: A quantitative comparison of multiple access control protocols for wireless atm. IEEE Transactions on Vehicular Technology 50 (2001) 796 $-81549$

3. Kwok, Y.K., Lau, V.: Performance evaluation of multiple access control schemes for wireless multimedia services. IEE Proc - Communications 148 (2001) 86-94 49

4. Acampora, A.: Wireless atm: A perspective on issues and prospects. IEEE Personal Communication 3 (1996) 8-17 [4.]

5. Raychaudhuri, D.: Wireless ATM networks: Architecture, system design and prototyping. IEEE Personal Communication 3 (1996) 42-49 49

6. Ayanoglu, E., Eng, K.Y., Karol, M.J.: Wireless ATM: Limits, challenges, and proposals. IEEE Personal Communication 3 (1996) 18-36 49

7. Zheng, B., Atiquzzaman, M.: Traffic management of multimedia over ATM networks. IEEE Communications Magazine 37 (1999) 33-38 50,52

8. Batra, P., Chang, S.F.: Effective algorithms for video transmission over wireless channels. Signal Processing: Image Communication 12 (1998) 147-166 50]

9. Zhang, J., Frater, M.R., Arnold, J.F., Percival, T.M.: MPEG-2 video services for wireless ATM networks. IEEE Journal on Selected Areas in Communication 15 (1997) 119-128 50]

10. Sun, H., Zdepski, J.W., Kwok, W., Raychaudhuri, D.: Error concelment algorithms for robust decoding of MPEG. Signal Processing: Image Communication 10 (1997) 249-268 [50]

11. Zheng, B., Atiquzzaman, M.: Multimedia over high speed networks: reducing network requirement with fast buffer fillup. In: IEEE GLOBECOM'98, Sydney (1998) 779-784 55] 